\title{
The Effects of Benzene Substituents and Intermediate Linkage on Local Anesthetic Effectiveness
}

\author{
Vikas L Bommineni \\ University of Pennsylvania \\ bvikas@sas.upenn.edu
}




\begin{abstract}
There are many experimental studies on the effectiveness of clinically effective local anesthetics (LA). Although an atomic analysis of LAs would contribute to understanding the factors that influence their effectiveness, there have been no theoretical studies performed thus far. Previous prominent papers in the field have examined the aromatic ring and amine terminal of LAs in great detail. In this paper, I establish which type of intermediate linkage - ester or amide - lends to a relatively greater effectiveness. I also elucidate the extent to which substitutions on the LA's benzene ring differentially influence amino esters' and amino amides' relative effectiveness. Using novel structure-based drug design (SBDD) techniques, I observed that LA effectiveness is influenced more by benzene ring substitutions and, to a lesser degree, by the type of intermediate linkage.
\end{abstract}

\title{
Introduction
}

In general, there are two categories of anesthetics: local and general. A local anesthetic (LA) is a pharmaceutical drug that causes a temporary loss of pain sensation in a specific area or localized area of a person's body ${ }^{1}$. It also helps to treat epilepsy and cardiac arrhythmia as well.

Derived synthetically from cocaine, LAs have numbing properties that are especially useful in the fields of dentistry and surgery. These numbing properties are directly linked to the LAs molecular structure. Thus, it is important to comprehend the subtleties and moieties of their molecular structure as it plays a crucial role in the anesthetic pathway of action.

\section{Molecular structure}

All LAs are weak bases, membrane-stabilizing, and share a common structural form: an aromatic ring, an ionizable amine group, and an intermediate linkage group ${ }^{2,3}$. More specifically, LAs are separated into 2 categories: amino esters and amino amides. The intermediate linkage group determines the LA's identity as either an amide or ester; however, the linkage group's potential as a determinant of LA action has not been researched yet. Most research has focused on the pivotal roles of the aromatic ring and the amine terminal in drug mechanism ${ }^{4}$.

\section{LA mechanism: A general overview}

How does this mechanism work? According to Hille's modulated receptor hypothesis ${ }^{5}$, all LAs attach to a common receptor on voltage-gated sodium $(\mathrm{Na}+)$ channels in nerve cell membranes, thereby temporarily preventing the propagation of action potentials along nerve cells. As such, the LA acts as a signal antagonist ${ }^{6}$ and prevents the overstimulation of signal impulses associated with epilepsy, arrhythmia, and pain sensation ${ }^{3}$.

However, the exact dynamics of LAs remain mostly unclear ${ }^{7}$. Because of a lack of structural data on membrane proteins, nobody is sure whether LAs exert their effects directly on the $\mathrm{Na}+$ 
channels or indirectly on the surrounding phospholipids ${ }^{8}$. Furthermore, the asymmetry and the membrane's variable voltage has made following the LA pathway more difficult ${ }^{9}$. As a result, the mechanism has been debated since the initial discovery of the receptor almost forty years $\operatorname{ago}^{3,10}$.

Some thought that the LA interacted with the membrane phospholipids ${ }^{11}$, while others thought they interacted with the transmembrane proteins ${ }^{12}$; however, more recent literature used the amino acid residues of the $\mathrm{Na}+$ channel to elucidate the exact molecular structure of the channel and receptor site ${ }^{13,14}$. In the past decade, scientists have shifted focus towards this binding site for the tertiary LA located in the pore of the $\mathrm{Na}+$ channel $^{9,14,15}$. The receptor lies in the S6 segment of domain $\mathrm{IV}^{16}$ of the $\mathrm{Na}+$ channel, which is only accessible from the intracellular, cytoplasmic entrance of said channel. In other words, it's only accessible by the non-ionized form of a LA ${ }^{9}$. This crucial form is also known as the tertiary form due to the structure of the LA amine terminal. The modern hypothesis of LA action suggests that the local anesthetic molecules must be transferred from solution to the hydrophobic environment of biological membranes to give rise to anesthetic action ${ }^{4}$. Consequently, the tertiary LA plays a crucial role in this mechanism.

\section{Modern hypothesis of local anesthetic action}

Specifically, the most widespread hypothesis depicts tertiary LA molecules penetrating deep inside the lipid bilayer of the nerve cell membrane ${ }^{6}$. Once inside, the amine group is ionized and subsequently attaches to the $\mathrm{Na}^{+}$channel binding site in a process called "ion-trapping". Consequently, LA molecules can't leave the membrane until they become unionized.

As a special exception, a neutral LA can attach to the binding site without becoming protonated, but when it does, dissociation from the channel is nearly instantaneous ${ }^{4,17}$. Meanwhile, the ionized LA molecules, or quaternary molecules, attach to the vast array of other extracellular transmembrane proteins, altering toxicity and voltage in the structurally asymmetric phospholipids $^{18}$.

Stronger binding of the quaternary LA to the sodium channel's binding site protects the LA from blood clearance ${ }^{9}$. As a consequence, the "effectiveness" of a LA relies on the chemical makeup of the tertiary LA.

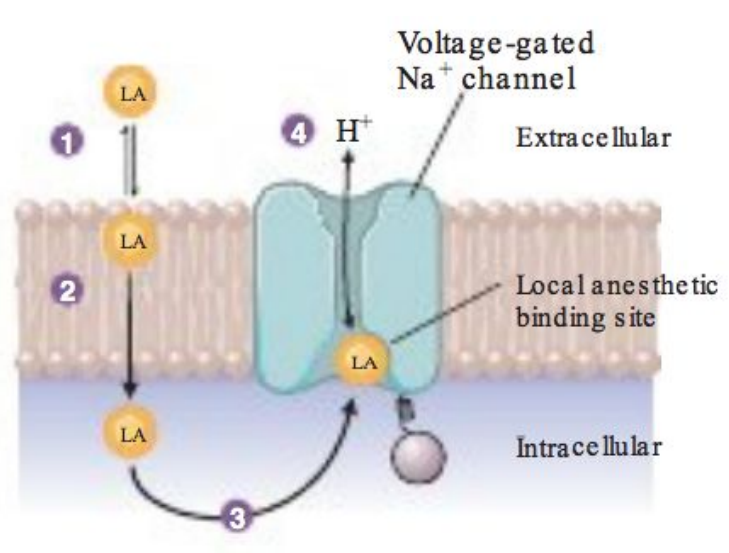

Figure $\mathbf{1}^{19}$ (on the right): A visual depiction of the modern hypothesis. 1) The tertiary LA enters and 2) diffuses through the phospholipid bilayer membrane. 3) Tertiary LA becomes ionized and attaches to a common binding site as a quaternary LA.

\section{LA Dissociation in Aqueous Solution}

Regardless of ester or amide linkage, the LA is introduced to the human physiologic environment as a water-soluble hydrochloride salt ${ }^{4}$.

Consequently, the LA exists in an equilibrium between its nonionized form and cationic form in 
aqueous solution: Tertiary $\mathrm{LA}+\mathrm{H}_{2} \mathrm{O}^{+} \leq$Quaternary $\mathrm{LA}+\mathrm{OH}^{-}$(Eqn. 1). Assuming a physiologic $\mathrm{pH}$ of $\sim 7.4^{20}$, the exact proportion of quaternary to tertiary LAs in the physiologic environment can now be computationally determined via the modified Henderson-Hasselbach equation ${ }^{21}: 6.6-p K b=\log \left(\frac{\text { Quaternary } L A}{\text { Tertiary } L A}\right)$ (Eqn. 2). The “ $\mathrm{pK}_{\mathrm{b}}$ " in Eqn. 2 refers to the equilibrium base constant, or the quantitative measure of the strength of a base in aqueous solution. For LAs, their $\mathrm{pK}_{\mathrm{b}}$ values range from 5 to 6.5 , which are relatively low values for a drug ${ }^{22}$. Nevertheless, this constant is crucial to the anesthetic mechanism since it determines the presence of tertiary LAs, which directly influence the LA's duration of action.

\section{Effectiveness of a LA}

How does a greater concentration of tertiary molecules increase LA duration? The answer lies in the effectiveness of an LA. Effectiveness is not the same as efficacy. All LAs have relatively similar efficacies; however, they vary in duration and time of onset ${ }^{2}$. In the non-life threatening situations that call for the use of local anesthetics, the LA needs to keep a patient under anesthesia as long as possible; the time of onset is relatively unimportant. As such, the duration of action of a LA has been identified as most critical to a drug's effectiveness.

In order to determine the duration of action, it is necessary to look at how the following changes can influence the anesthetic mechanism:

Dissociation and the $p K_{b}$ value

Since only the tertiary forms can penetrate the nerve cell membrane and bind to the receptor on the $\mathrm{Na}+$ channel, they alone are responsible for the initiation and continuation of the LA's effects ${ }^{4,9}$. Thus, a greater ratio of tertiary LA molecules to quaternary LA molecules will result in a longer duration of action. And, as mentioned earlier, this ratio can be determined using Eqn. 2. Thus, a more basic LA with an associated higher $\mathrm{pK}_{\mathrm{b}}$ corresponds to a greater amount of tertiary molecules present in the environment; consequently, a higher $\mathrm{pK}_{\mathrm{b}}$ value leads to a longer duration of action.

\section{Aliphatic substitutions of the aromatic ring}

The more lipophilic the LA, the easier the tertiary LA can penetrate the membrane ${ }^{4,9}$. As current literature shows ${ }^{2}$, only the aromatic ring is known to definitely affect the lipid solubility of the entire molecule. More specifically, direct aliphatic substitutions of the aromatic ring are known to contribute to increased lipophilicity and thus greater membrane penetration. With more tertiary LA molecules able to penetrate the neuronal sheath, there is an associated increase in duration of action. 
After the LA has entered the membrane interior and become protonated, it needs to attach to the sodium channel receptor. A larger partial charge on the amine terminal corresponds to a greater binding affinity to the receptor, preventing clearance of the $\mathrm{LA}^{2,9,14}$. Hypothetically ${ }^{21}$, substitutions to the LA amide terminal or aromatic ring should increase partial charge magnitude. Also, the quaternary forms of the LA are able to bind tightly to the extracellular membrane proteins, preventing blood clearance of the $\mathrm{LA}^{23}$. Regardless of how the partial charge was changed, a stronger partial charge allowed more of the LA to remain in the cell, increasing the LA's duration of action.

Taking these properties collectively, the relative duration of actions between different LAs can theoretically be compared ${ }^{4}$.

\section{Limitation of effectiveness}

LAs can be produced relatively cheaply and easily compared to other drugs ${ }^{20}$. Also, the duration of action could theoretically be increasing lipophilicity and partial charge. However, there is a limiting factor to how effective a LA can be: the toxicity of the LA ${ }^{20}$. Otherwise, LAs could be designed to last for longer periods of time. For example, a special group of LA amides with a ring conformation about the amine terminal called pipecoloxylidide have similar durations to amino esters. However, the cardiotoxicity of LAs in this group are too high to be used for clinical purposes ${ }^{20}$.

By comparing these intermediate linkage groups to each other, it is possible to gain more insight into the longer duration of block for amino ester compared to amino amides.

\section{Qualitative observation on ester and amide linkages}

However, with respect to duration of action, the role of the ester and amide linkage has not yet been discussed in literature. This is especially surprising given the vast collection of data on the two types of LAs. Furthermore, identically bonded amino esters and amino amides have displayed a large difference in effectiveness ${ }^{23}$.

Their difference in effectiveness is demonstrated by the absence of amino amides on the most recently published edition of the World Health Organization's (WHO's) Most Essential Medications List ${ }^{8}$. And this is for good reason, as amino amides have duration of actions of under 2 hours while amino esters have duration of actions between 2 to 8 hours ${ }^{24}$. This list publishes "the most efficacious, safe and cost-effective medicines for priority conditions". In other words, the LAs on this list are the most primed to be used in a clinical setting.

Yet, this list also suggests that amide-linkage confers a disadvantage to the effectiveness of a LA when compared to an ester-linkage. 


\section{Methodology}

To compare whether an ester or amide linkage leads to greater effectiveness, it is essential to calculate the 2 key properties determining a LA's duration of action: protonation propensity and binding potential. To help further determine which linkage is more effective, it is necessary to also ask how the aromatic ring's substituents' effects on the key properties vary depending on the linkage; this will allow for a more realistic analysis since most clinically effective LAs have substituted groups on the benzene ring.

These core calculations are best done by analyzing changes in the 2 key properties for both types of LAs before and after "R group" substitutions on the aromatic ring. Since every LA follows the core structural form seen in Picture 1, any substitution(s) of any atoms(s) at the R position(s) that leads to changes in the properties will have occurred as a direct result of the substitution(s). Using my "rudimentary LAs" (RLAs) in Picture 2 as the standard, the exact changes in the two properties can linked to the specific intermediate linkage and substitution changes. For example, a rudimentary amino ester (RAE) and rudimentary amino amide (RAA) were identically methylated at the same $\mathrm{R}$ position. These same molecules were ionized, and substituted. Then, the relevant calculations were made for both the tertiary and quaternary forms. From this process, I was able to see how having an ester and amide linkage affected the changes in the two key effectiveness properties. This technique is derived based on the principles of $\mathrm{SBDD}^{25}$.

Using the GAMESS quantum chemistry program, DFT/B3LYP vibrational frequency and geometry optimization calculations with the 6-31G** basis set were performed for RLAs and their substituted derivatives; identical calculations were done for their respective ionized forms. Due to time constraints, substitutions will only be made on the benzene ring and not on the amide terminal. In particular, calculations for the ionized and unionized single methyl RLAs, double methyl RLAs, chlorinated RLAs, and RLAs were performed. Chlorine and methyl were chosen as the substituted groups at specific $\mathrm{R}$ positions since they were the most prevalent in clinically-useful applications.

On a side note, a discussion of the $\mathrm{pK}_{\mathrm{b}}$ values' effect on LA effectiveness has been done previously and would only mimic previous results; however, it is necessary to acknowledge that $\mathrm{pK}_{\mathrm{b}}$ still determines the concentration of quaternary and tertiary molecules in the psychologic environment. 

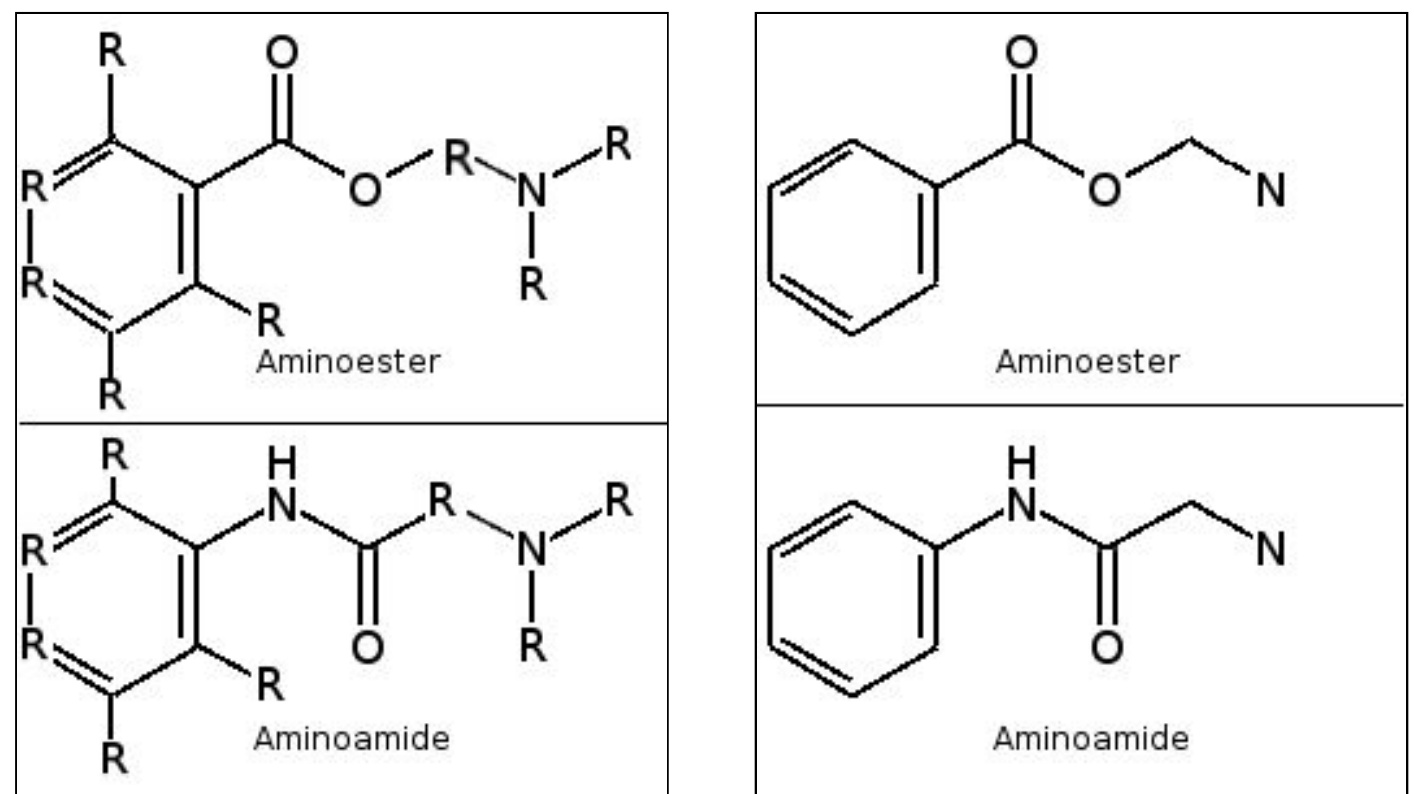

Figure 1 (on the left): The standard structural formulas of all amino esters and amino amides. Note: the furthest right nitrogen atom is commonly referred to as the "N-terminal"16.

Figure 2 (on the right): A rudimentary amino ester and a rudimentary amino amide; Note: for the amino ester, the ester oxygen points towards the benzene ring; for the amino amide, the amide nitrogen points away from the benzene ring. This is due to the choice of using only the L-enantiomer.

\section{Testing for Protonation Propensities}

To assess which intermediate linkage leads to greater protonation propensity, I obtained the zero-point energy (ZPE) corrected free energies from the raw output of vibrational frequency calculations for each tested $\mathrm{LA}_{1}, \mathrm{LA}_{2}, \mathrm{LA}_{1}^{+}$, and $\mathrm{LA}_{2}{ }^{+}$. Using Eqn. 1 and assuming an ideal physiologic environment, these local anesthetics exist in an equilibrium such that,

$$
\begin{aligned}
& \mathbf{L A}_{1}(\mathrm{aq})+\mathbf{H}^{+}(\mathrm{aq}) \leftrightharpoons \mathbf{L A}_{1}^{+}(\mathrm{aq})(\text { Eqn. 3) } \\
& + \\
& \mathbf{L A}_{2}(\mathrm{aq})+\mathbf{H}^{+}(\mathrm{aq}) \leq \mathbf{L A}_{2}^{+}(\mathrm{aq})(\text { Eqn. } 4)
\end{aligned}
$$

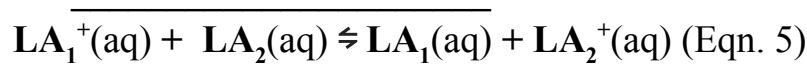

Using ZPE corrected free energies, I calculated the Gibbs free energies for each molecule in Eqn. 5.

For any aqueous equilibrium reaction in the form of Eqns. 3, 4, or 5:

$\Delta G_{\text {aq }}=\Sigma\left(\mathbf{G}+\mathbf{G}_{\text {corr }}\right)_{\text {products }}-\boldsymbol{\Sigma}\left(\mathbf{G}+\mathbf{G}_{\text {corr }}\right)_{\text {reactants }}$ (Eqn. 6), where $\mathrm{G}_{\text {corr }}$ is the ZPE correction for the vibrational energy that persists at $0 \mathrm{~K}$. 
Thus, the $\Delta G_{\text {aq }}$ with a distinct positive or negative sign can be calculated for Eqn. 5. If $\Delta G_{\text {aq }}$ is negative, then the forward reaction is more favorable. In other words, $\mathrm{LA}_{2}$ will be more likely to be found protonated than $\mathrm{LA}_{1}$. However, if $\Delta G_{\text {aq }}$ is positive, then the reverse reaction is more favorable, and $\mathrm{LA}_{1}$ is more likely to be found protonated than $\mathrm{LA}_{2}$.

As a result, LA molecules with different intermediate linkages can be compared in terms of protonation propensity.

\section{Testing for Binding Potential}

To assess the binding potential of a LA to the Hillean-predicted common receptor site, I used the raw outputs from the step above to collect the N-terminal $\left(\mathrm{N}_{t}\right)$ partial charges for all tested LAs. A more negative $\mathrm{N}_{\mathrm{t}}$ atom will lead to a stronger bond to the positively charged receptor region $^{9,26}$ in the S6 segment; although, it is important to note that this is only based on a hypothesis. In actuality, many other factors affect the LA's duration of action.

\section{Assumptions}

To apply these computations in a relevant way requires 6 key simplifications to the mechanism of action of a LA.

1) The biggest assumption is that the membrane would consist of a set of homogenous phospholipids. Evidently ${ }^{4,9,14,27}$, this is not true. In terms of lipid compositions, the phospholipid bilayer is better thought of as two asymmetric monolayers ${ }^{28}$. Additionally, the binding of the ionized LA to the transmembrane proteins and surrounding lipids further morphs the structure of the phospholipid bilayer. Thus, the membrane is rarely stable, and the tertiary LA molecule will experience a barrier to membrane penetration.

2) It is assumed that the voltage-gated sodium channel will not exhibit state-dependent blockade $^{9,28}$. This means that the sodium channel will always be activated and open to more tertiary LAs as long the sodium channel's receptor is open; however, in reality, LAs can only bind to the receptor during membrane depolarization when the sodium channel is open.

3) My methodology assumes that the drug will be introduced in the absence of its antagonists or other complications; however, this isn't necessarily true.

4) Apart from toxicity, molecular size is also limited physically ${ }^{28}$. The phospholipid bilayer is unable to diffuse molecules that are too large in size. In this computation, however, the LAs are similar in size to molecules used in other membrane-based computation papers to avoid that complication .

5) We assume that only the L enantiomer of any LA is used. Without this assumption, the absorption, distribution, potency, and therapeutic action would be affected by racemic mixtures of the $\mathrm{D}$ and $\mathrm{L}$ enantiomers ${ }^{29}$.

6) All LAs and their ionized forms are compared in identical concentrations. In most clinical experiments, the $\mathrm{pK}_{\mathrm{b}}$ determines the ratio of quaternary to tertiary molecules in the 
environment. By assuming identical concentrations of tertiary and quaternary LAs, $\mathrm{pK}_{\mathrm{b}}$ values can be ignored.

\section{Results}

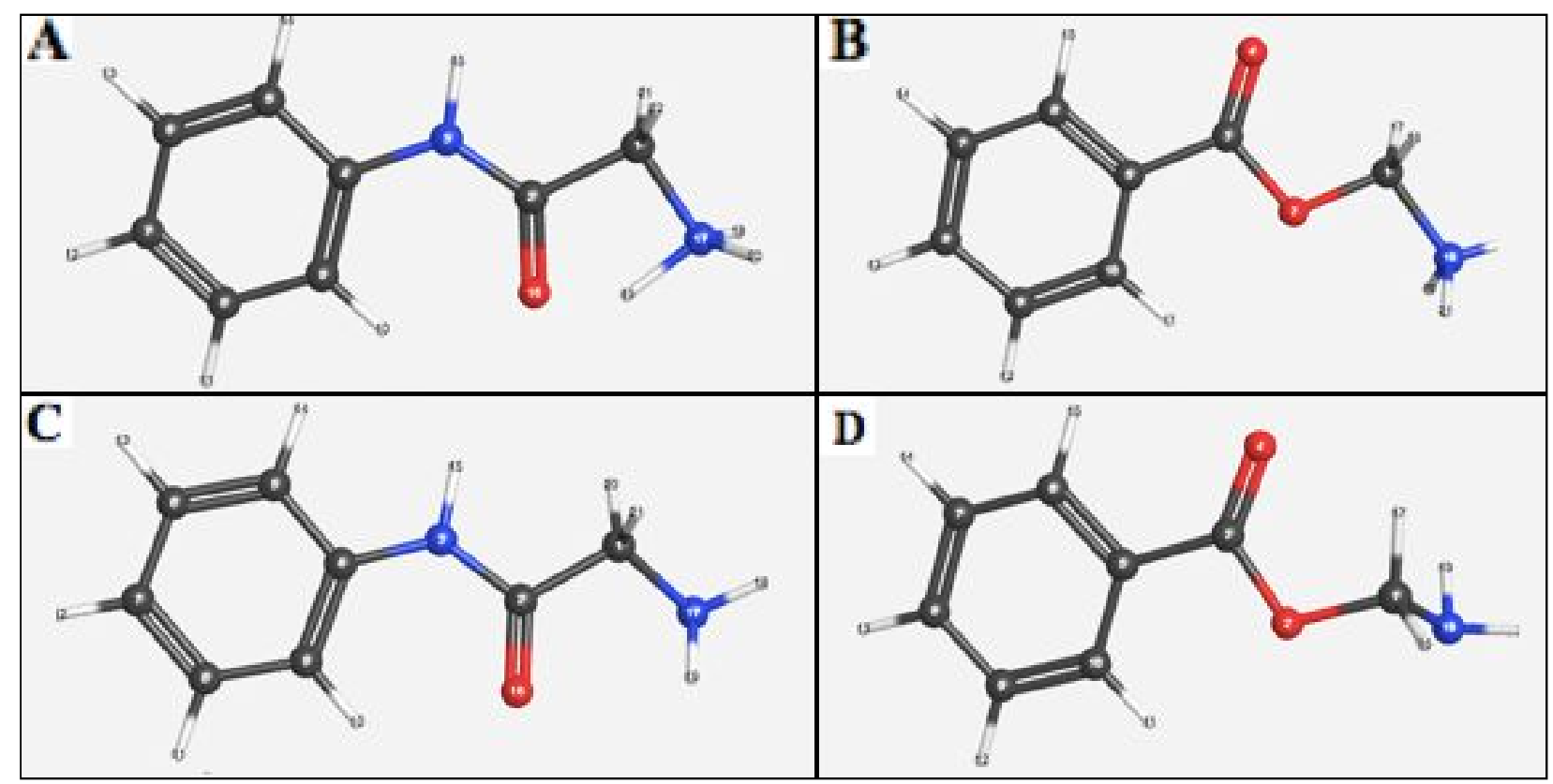

FIG. 3 (above). A and C both represent the RAA, ionized and unionized respectively. B and D both represent the RAE, ionized and unionized respectively.

TABLE 1. The RLAs: RAE and RAA. Calculated thermochemistry values from GAMESS for Eqn. 5, where both the protonated and unprotonated LAs are the RAE and the RAA.

\begin{tabular}{|c|c|c|c|c|}
\hline & $\operatorname{RAE}\left[\mathbf{L A}_{1}\right](\mathbf{a q})$ & $\begin{array}{l}\text { Ionized RAE } \\
{\left[\mathbf{L A}_{1}^{+}\right] \text {(aq) }}\end{array}$ & $\operatorname{RAA}\left[\mathrm{LA}_{2}\right](\mathbf{a q})$ & $\begin{array}{l}\text { Ionized RAA } \\
{\left[\mathbf{L A}_{2}^{+}\right] \text {(aq) }}\end{array}$ \\
\hline \multirow[t]{2}{*}{$\mathbf{E}_{0}+\mathbf{G}_{\mathrm{corr}}$} & & & & \\
\hline & $782.35 \mathrm{~kJ} / \mathrm{mol}$ & $864.13 \mathrm{~kJ} / \mathrm{mol}$ & $874.97 \mathrm{~kJ} / \mathrm{mol}$ & $937.89 \mathrm{~kJ} / \mathrm{mol}$ \\
\hline $\begin{array}{l}\text { Change in Gibbs } \\
\text { energy for } \\
\text { equilibrium } \\
\text { reaction ( } \Delta G_{\text {aq }} \text { ) } \\
\text { [for Eqn. 5] (in } \\
\text { kJ/mol) }\end{array}$ & $-18.8565 \mathrm{~kJ} / \mathrm{mol}$ & $-18.8565 \mathrm{~kJ} / \mathrm{mol}$ & $-18.8565 \mathrm{~kJ} / \mathrm{mol}$ & $-18.8565 \mathrm{~kJ} / \mathrm{mol}$ \\
\hline $\begin{array}{l}N_{t} \text { partial charge } \\
\text { (in } e \text {-) }\end{array}$ & -0.389388 & -0.328914 & -0.425592 & -0.350682 \\
\hline
\end{tabular}




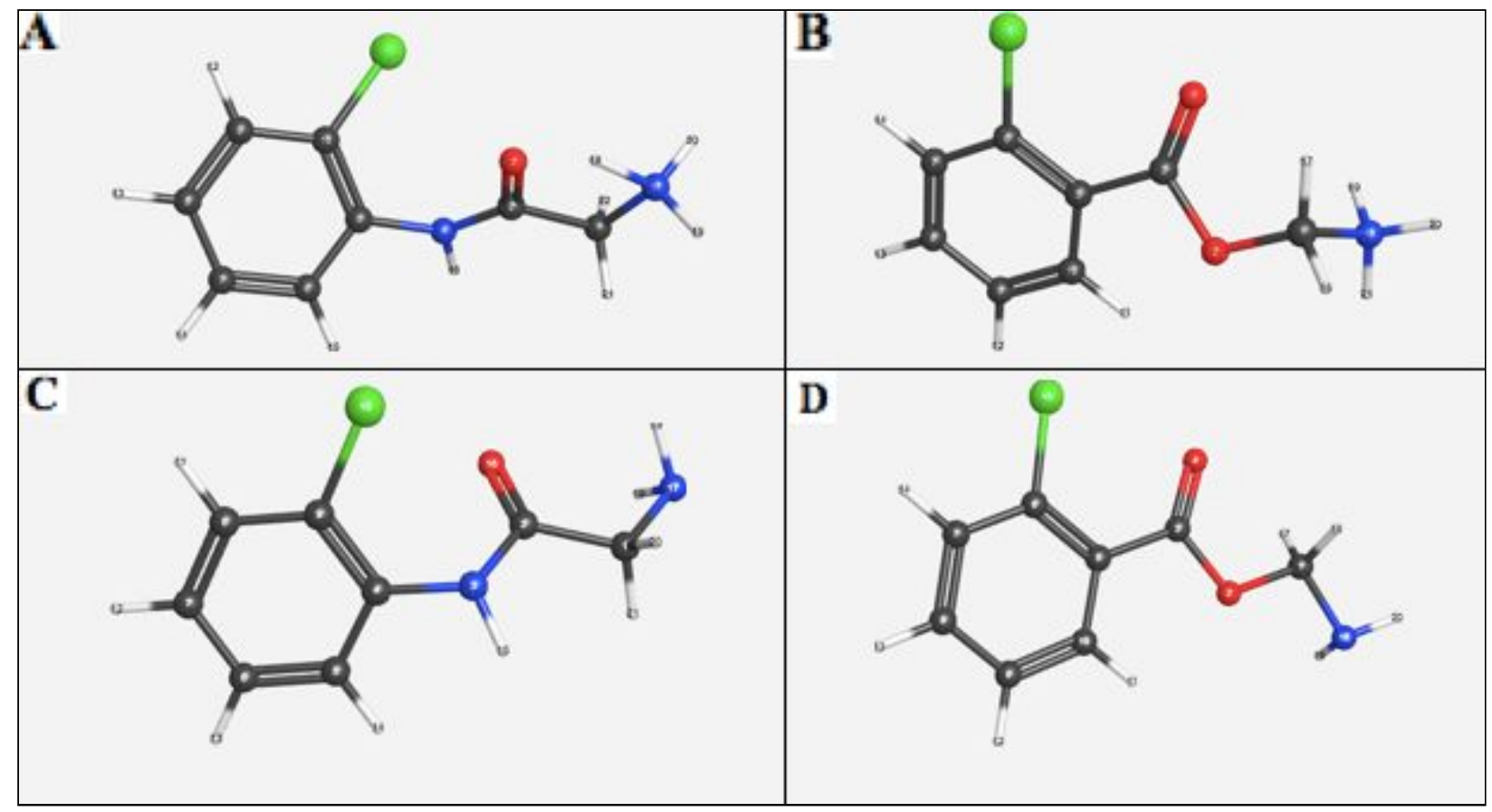

FIG. 4 (above). A and C both represent the chlorinated RAA, ionized and unionized respectively. $B$ and D both represent the chlorinated RAE, ionized and unionized respectively. Note: the chlorine substitution occur on the same carbon for all the RAAs and RAEs.

TABLE 2. The chlorinated RLAs: Chlorinated RAE And RAA. Calculated thermochemistry values from GAMESS for Eqn. 5, where the RLAs are both chlorinated in their protonated and unprotonated forms.

\begin{tabular}{|c|c|c|c|c|}
\hline & $\operatorname{RAE}\left[\mathrm{LA}_{1}\right](\mathrm{aq})$ & $\begin{array}{l}\text { Ionized RAE } \\
{\left[\mathbf{L A}_{1}^{+}\right](\mathbf{a q})}\end{array}$ & $\operatorname{RAA}\left[\mathbf{L A}_{2}\right](\mathbf{a q})$ & $\begin{array}{l}\text { Ionized RAA } \\
{\left[\mathbf{L A}_{2}^{+}\right] \text {(aq) }}\end{array}$ \\
\hline \multirow[t]{2}{*}{$\begin{array}{l}E_{0}+G_{\text {corr }}(\text { in } \\
\mathrm{kJ} / \mathrm{mol})\end{array}$} & & & & \\
\hline & $723.650 \mathrm{~kJ} / \mathrm{mol}$ & $801.937 \mathrm{~kJ} / \mathrm{mol}$ & $794.134 \mathrm{~kJ} / \mathrm{mol}$ & $876.822 \mathrm{~kJ} / \mathrm{mol}$ \\
\hline $\begin{array}{l}\text { Change in Gibbs } \\
\text { energy for } \\
\text { equilibrium } \\
\text { reaction ( } \Delta G_{\text {aq }} \text { ) } \\
\text { [for Eqn. 5] (in } \\
\text { kJ/mol) }\end{array}$ & $+4.401 \mathrm{~kJ} / \mathrm{mol}$ & $+4.401 \mathrm{~kJ} / \mathrm{mol}$ & $+4.401 \mathrm{~kJ} / \mathrm{mol}$ & $+4.401 \mathrm{~kJ} / \mathrm{mol}$ \\
\hline $\begin{array}{l}\mathrm{N}_{\mathrm{t}} \text { partial charge } \\
\text { (in } e \text {-) }\end{array}$ & -0.386793 & -0.331363 & -0.382514 & -0.350619 \\
\hline
\end{tabular}




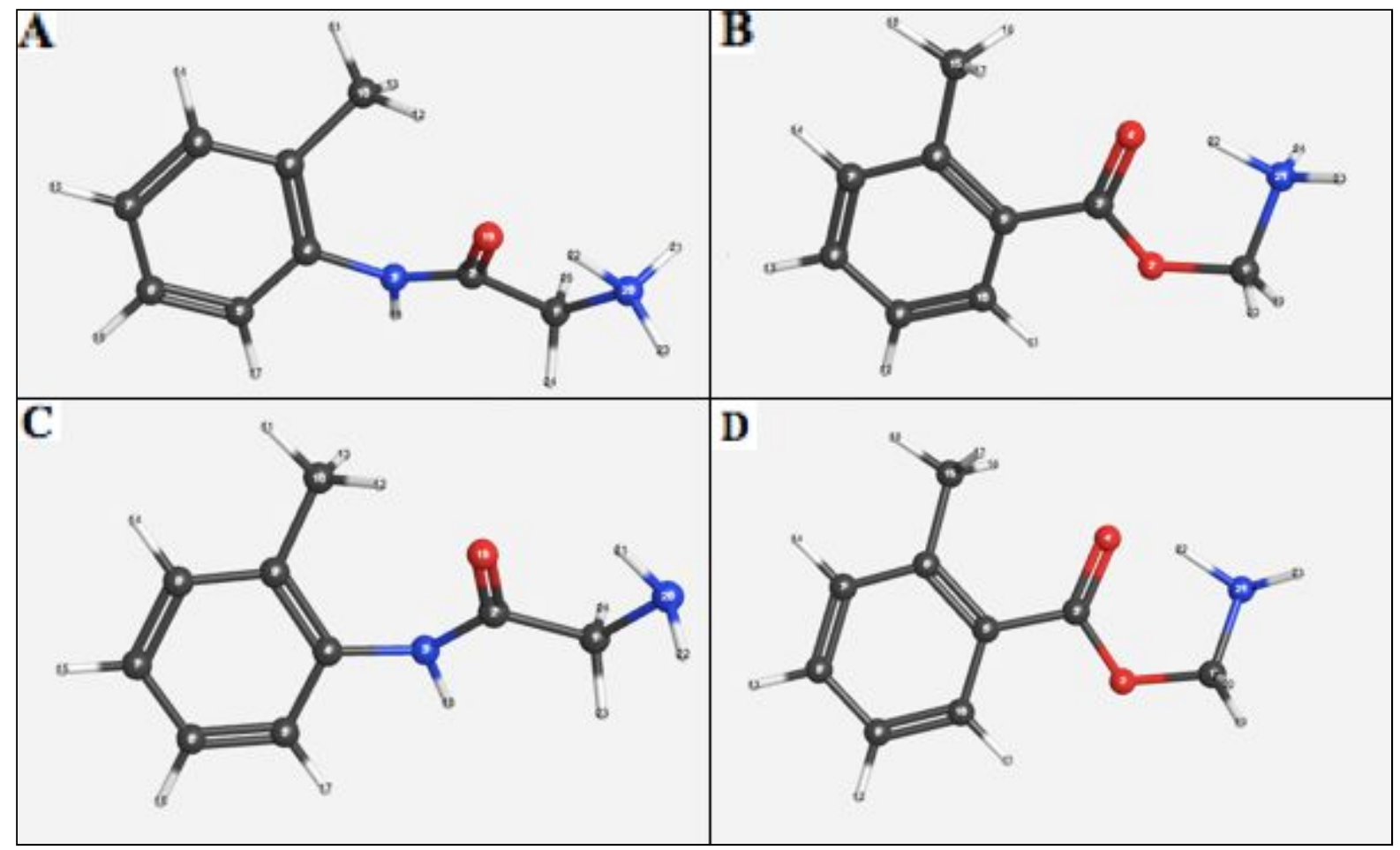

FIG. 5 (above). A and C both represent the single methyl RAA, ionized and unionized respectively. B and D both represent the single methyl RAE, ionized and unionized respectively. Note: the methyl group substitution occurs on the same carbon for all the RAAs and RAEs.

TABLE 3. The single methyl RLAs: Single Methyl RAE And RAA. Calculated thermochemistry values from GAMESS for Eqn. 5, where the RLAs are both methylated in their protonated and unprotonated forms.

\begin{tabular}{|c|c|c|c|c|}
\hline & $\operatorname{RAE}\left[\mathrm{LA}_{1}\right](\mathrm{aq})$ & $\begin{array}{l}\text { Ionized RAE } \\
{\left[\mathbf{L A}_{1}^{+}\right] \text {(aq) }}\end{array}$ & $\operatorname{RAA}\left[\mathbf{L A}_{2}\right](\mathbf{a q})$ & $\begin{array}{l}\text { Ionized RAA } \\
{\left[\mathbf{L A}_{2}^{+}\right] \text {(aq) }}\end{array}$ \\
\hline \multirow[t]{2}{*}{$\begin{array}{l}E_{0}+G_{\text {corr }} \text { (in } \\
\mathrm{kJ} / \mathrm{mol})\end{array}$} & & & & \\
\hline & $947.896 \mathrm{~kJ} / \mathrm{mol}$ & $1,025.14 \mathrm{~kJ} / \mathrm{mol}$ & $1,021.12 \mathrm{~kJ} / \mathrm{mol}$ & $1,100.16 \mathrm{~kJ} / \mathrm{mol}$ \\
\hline $\begin{array}{l}\text { Change in Gibbs } \\
\text { energy for } \\
\text { equilibrium } \\
\text { reaction ( } \Delta G_{\mathrm{aq}} \text { ) } \\
\text { [for Eqn. 5] (in } \\
\mathrm{kJ} / \mathrm{mol} \text { ) }\end{array}$ & $+1.796 \mathrm{~kJ} / \mathrm{mol}$ & $+1.796 \mathrm{~kJ} / \mathrm{mol}$ & $+1.796 \mathrm{~kJ} / \mathrm{mol}$ & $+1.796 \mathrm{~kJ} / \mathrm{mol}$ \\
\hline $\begin{array}{l}\mathrm{N}_{\mathrm{t}} \text { partial charge } \\
\text { (in } e \text {-) }\end{array}$ & -0.394293 & -0.359283 & -0.394564 & -0.351425 \\
\hline
\end{tabular}




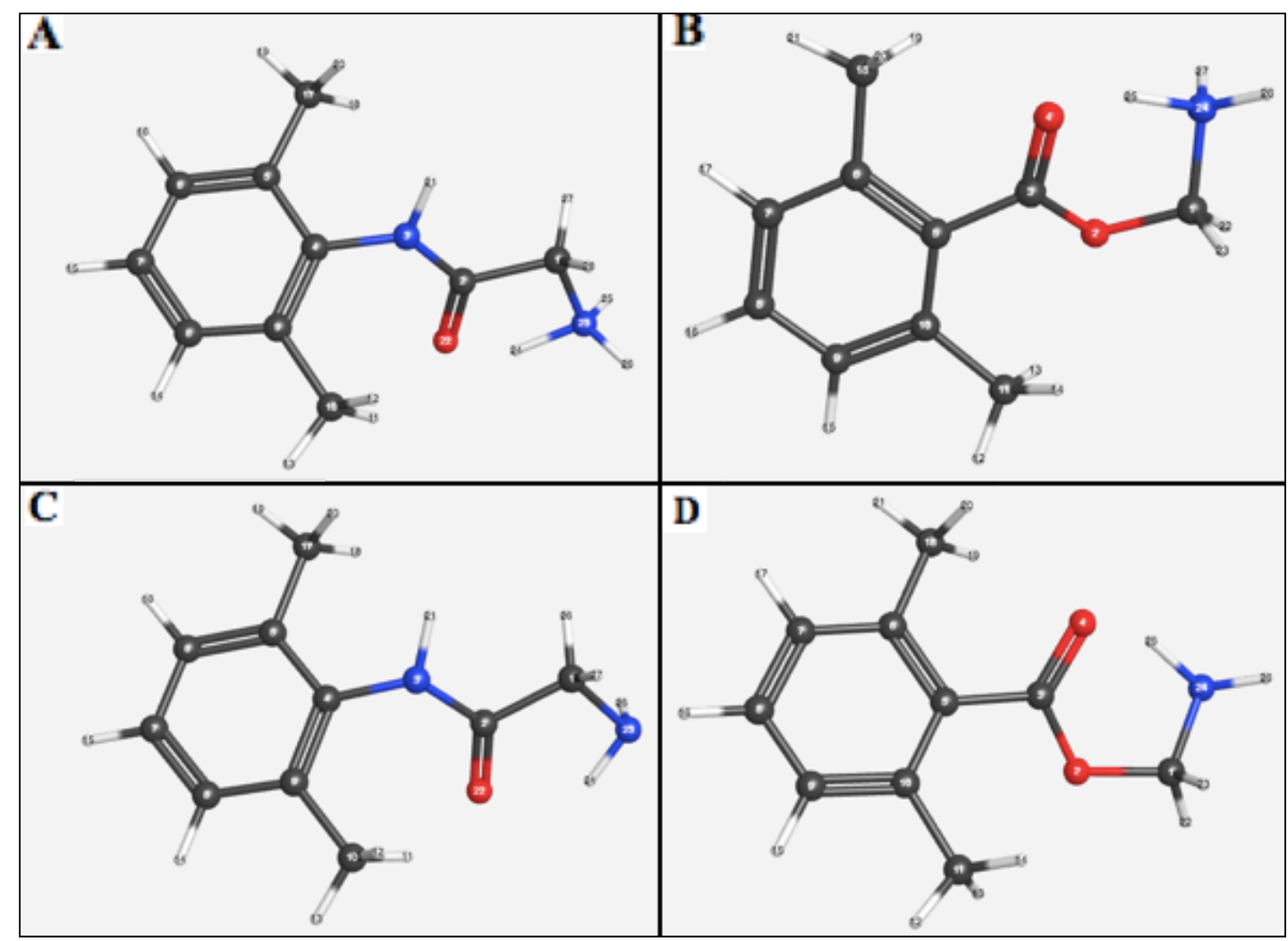

FIG. 5 (above). A and C both represent the double methyl RAA, ionized and unionized respectively. B and D both represent the double methyl RAE, ionized and unionized respectively. Note: the methyl group substitution occurs on the same carbon for all RAAs and RAEs.

TABLE 4. The double methyl RLAs: Double Methyl RAE and RAA. Calculated thermochemistry values from GAMESS for Eqn. 5, where the RLAs are both doubly methylated in their protonated and unprotonated forms.

\begin{tabular}{|c|c|c|c|c|}
\hline & $\operatorname{RAE}\left[\mathrm{LA}_{1}\right](\mathrm{aq})$ & $\begin{array}{l}\text { Ionized RAE } \\
{\left[\mathbf{L A}_{1}^{+}\right](\mathbf{a q})}\end{array}$ & $\operatorname{RAA}\left[\mathbf{L A}_{2}\right](\mathbf{a q})$ & $\begin{array}{l}\text { Ionized RAA } \\
{\left[\mathbf{L A}_{2}^{+}\right] \text {(aq) }}\end{array}$ \\
\hline \multirow[t]{2}{*}{$\begin{array}{l}\mathbf{E}_{0}+\mathbf{G}_{\text {corr }}(\text { in } \\
\mathbf{k J} / \mathbf{m o l})\end{array}$} & & & & \\
\hline & $1,101.91 \mathrm{~kJ} / \mathrm{mol}$ & $1,181.73 \mathrm{~kJ} / \mathrm{mol}$ & $1,176.39 \mathrm{~kJ} / \mathrm{mol}$ & $1,253.13 \mathrm{~kJ} / \mathrm{mol}$ \\
\hline $\begin{array}{l}\text { Change in Gibbs } \\
\text { energy for } \\
\text { equilibrium } \\
\text { reaction ( } \Delta G_{\mathrm{aq}} \text { ) } \\
\text { [for Eqn. 5] (in } \\
\mathrm{kJ} / \mathrm{mol} \text { ) }\end{array}$ & $-3.080 \mathrm{~kJ} / \mathrm{mol}$ & $-3.080 \mathrm{~kJ} / \mathrm{mol}$ & $-3.080 \mathrm{~kJ} / \mathrm{mol}$ & $-3.080 \mathrm{~kJ} / \mathrm{mol}$ \\
\hline $\begin{array}{l}N_{\mathrm{t}} \text { partial charge } \\
\text { (in } e_{-} \text {) }\end{array}$ & $\mathbf{- 0 . 3 9 3 3 5 9}$ & $-\mathbf{- 0 . 3 5 9 5 8 7}$ & $\mathbf{- 0 . 3 9 5 8 8 5}$ & $-\mathbf{0 . 3 5 1 7 5 7}$ \\
\hline
\end{tabular}




\section{Discussion}

The following extrapolated percentages and quantitative analyses are subject to negligible discrepancies due to rounding errors $(\sim .01 \%)$.

\section{Changes in Partial Charges}

In order to bind to the positively charged $\mathrm{Na}^{+}$channel receptor for LAs, a ionized LA needs to have a negative partial charge on its $\mathrm{N}_{\mathrm{t}}$. To apply these findings, I will be comparing the partial charges between different structures as well as between the ionized and pre-ionized form of the same structures. Unless explicitly stated otherwise, all expressed percent comparisons in this discussion are made in terms of partial charge magnitude. I chose to do this to simplify the following discourse since the $\mathrm{N}_{\mathrm{t}}$ partial charges of all RLAs became less negative after protonation and substitutions. Thus, the percent changes in $\mathrm{N}_{\mathrm{t}}$ partial charge indicates a move towards a smaller yet still negative partial charge.

\section{Linkage Effects in RLAs}

In all 4 tables, unionized RAE and its derivatives had weaker partial charges than unionized RAAs and its derivatives. From Table 1 , the $\mathrm{N}_{\mathrm{t}}$ partial charge was greater by $8.4 \%$ for the unionized RAEs. Meanwhile, on average, ionized RAEs had a 6.2\% greater partial charge than the ionized RAAs. The only difference between these compared groups is the intermediate linkage. Therefore, the ester linkage must be contributing to the greater average negative charge of the ionized RAEs' $\mathrm{N}_{\mathrm{t}} \mathrm{s}$. Thus, my observation of the WHO's Most Essential Medications List ${ }^{24}$ is confirmed: ester linkages lead to a more negative $\mathrm{N}_{t}$ partial charge than the amide linkage and thus longer durations. Yet, it is difficult to come to any definite conclusions based solely on this result. It is essential to examine the effects of the substitutions on the $\mathrm{N}_{\mathrm{t}}$ partial charge to see whether my observation persists.

\section{Substitution of one methyl group and its Effects}

When I compared my ionized single methyl RLAs to my ionized RLAs, the average $\mathrm{N}_{\mathrm{t}}$ partial charge was greater by $4.4 \%$ on the ionized single methyl RLAs, according to Table 3 . Thus, the addition of a single methyl group to the aromatic ring increases the ability of an LA to bond to the receptor site. This finding is novel, in that aromatic ring substitutions were previously thought to have negligible or nil effect in terms of binding potential and $\mathrm{N}_{\mathrm{t}}$ partial charge ${ }^{30}$. Comparing single methyl RLAs to RLAs, the single methyl ionized RAAs' $\mathrm{N}_{\mathrm{t}}$ partial charge decreased significantly more from the methyl substitution than the single methyl ionized RAEs' $\mathrm{N}_{\mathrm{t}}$ partial charges. More generally, using data from all 4 tables, the average percent decrease due to ionization was $8.88 \%$ for all RLAs with ester linkage; for RLAs with amide linkage, it was $10.9 \%$. Thus, it is seen that the type of linkage can affect the effect of aliphatic groups on $\mathrm{N}_{t}$ 
partial charge, and consequently $\mathrm{Na}^{+}$receptor binding potential. More specifically, the ester linkage is again favored as it experiences a smaller percent decrease in its $\mathrm{N}_{t}$ partial charge than the $\mathrm{N}_{\mathrm{t}}$ partial charge in amino amides.

\section{Substitution of two methyl groups and its effects}

However, since the vast majority of methylated clinical LAs have several methyl substitutions on their aromatic ring, it is more insightful to discuss when more than one methyl is added to the aromatic ring. The RLAs were also substituted with two methyl groups, as seen in Fig. 5. When comparing the RLAs to the single methyl RLAs, the average $\mathrm{N}_{\mathrm{t}}$ partial charge was $8.59 \%$ less smaller than the RLAs; for the amino amides, it was only $10.15 \%$ less than the RLAs. So, even though the $\mathrm{N}_{\mathrm{t}}$ partial charge became more positive in the single methyl RAA, the gap in percent change for the amides and esters decreases when yet another methyl group is added.

Hence, as expected, amino amides experience an easier transition to the neutral partial charge standard in the theoretical single methyl group model; however, as more methyl substitutions are made, the lower starting $\mathrm{N}_{\mathrm{t}}$ partial charge of amino esters can lead to a more positively charged $\mathrm{N}_{\mathrm{t}}$ compared to the amino amides' $\mathrm{N}_{\mathrm{t}}$. As a result, doubly substituted RAEs are sometimes predicted to have smaller durations of actions than doubly substituted RAAs. Thus, the observed WHO trend is broken in some scenarios. This anomaly in binding potentials can be explained through the pre-ionized $\mathrm{N}_{\mathrm{t}}$ partial charges. Since some RLAS have a smaller starting pre-ionized $\mathrm{N}_{t}$ partial charge than amino amides, they will end up having a $\mathrm{N}_{t}$ smaller negative charge after ionization and a smaller binding potential. This is probably why some amino esters i.e. chloroprocaine ${ }^{31}$ - experience shorter binding times and are less resistant to blood clearance.

\section{Substitution of chlorine and its effects}

Compared with the RLAs, singly chlorinated RLAs had smaller percent changes for both the amino esters (14.3\%) and amino amides (8.3\%) when ionized; however, the ionized chlorinated RLA had a greater percent change in the average $\mathrm{N}_{\mathrm{t}}$ partial charge than both cases of methylation. As a consequence, chlorination decreases the $\mathrm{N}_{\mathrm{t}}$ partial charge to a greater extent than single methylation. This is likely due to the fact that chlorine is an electrophile, and thus attracts electrons; through this proposed mechanism, additional chlorine substitutions would lead to a more positively charged $\mathrm{N}_{\mathrm{t}}$.

\section{Comparisons of Protonation Propensities}

Since amino esters are generally more effective, it would be reasonable to assume that they had longer durations of action; however, this is not true in all cases. In a few cases ${ }^{20,32}$, the amino amides had longer durations of action than amino esters. Still, for the most part, LAs with amide linkages were expected to be more favored for protonation in every derivative of the RLAs. 
However, some of the protonation propensity results don't agree with this idea. In these disagreeing scenarios, the partial charges of the LAs' $\mathrm{N}_{\mathrm{t}}$ and the LAs' $\mathrm{pK}_{\mathrm{b}}$ must have greater precedence than the protonation propensity of the LAs. Nevertheless, despite some calculated anomalies, protonation propensity is still a critical aspect of the LA mechanism and necessitates further analysis.

\section{Linkage Effects in RLAs}

Based on the $\Delta G_{\mathrm{aq}}$ from Table 1, the RAA was more likely to be found protonated than the RAE. Therefore, my results showed that LAs with an amide linkage have fewer tertiary molecules available to pass through the phospholipid bilayer membrane than identical LAs with an ester linkage. As a result, amino esters have more opportunities to interact with the binding receptor on the $\mathrm{Na}^{+}$channel. This result confirmed the WHO trend, where ester linkages have longer durations of actions; however, methyl substitution on the aromatic ring didn't continue the trend.

\section{Substitution of One Methyl Group Effect}

As the $\Delta G_{\mathrm{aq}}$ in Table 3 indicated, the single methyl RAE was more likely to be found protonated than the single methyl RAA. This led to the conclusion that the introduction of a methyl group onto a LA with ester linkage will favor protonation more so than if it were introduced onto an identical LA with amide linkage. The single methyl RLAs' protonation propensities disagreed with the WHO trend.

This single methyl substitution is a conundrum. Since the single methyl RLAs resemble clinically significant LAs more closely than the unsubstituted RLAs, the single methyl RLAs' results should take precedence. However, my results for double methyl RLAs agreed with the WHO trend.

\section{Substitution of Two Methyl Groups Effect}

As the $\Delta G_{\text {aq }}$ in Table 4 indicated, the double methyl RAA was more likely to be found protonated than the double methyl RAE. By adding another methyl group to the single methyl RAE, the amino ester is no longer favored for protonation. These substituted RLAs point to a LA with amino ester and two methyl substitutions having more tertiary molecules. The results have conformed to expectations.

With this contradictory data, it is impossible to come to a definite conclusion about which linkage group is preferred for protonation propensity when methyl groups are substituted onto the benzene ring. 


\section{Substitution of Chlorine Effect}

A single substitution of chlorine onto the RLAs' benzene rings yielded unexpected results. Based on the $\Delta G_{\mathrm{aq}}$ from Table 2, the chlorinated RAE was more likely to be found protonated than the chlorinated RAA. This result challenges the WHO trend that amide linkages yield a greater protonation propensity in all types of LAs.

However, this situation is different from the single methyl RLAs' situation presented earlier. There does exist some clinically useful LAs with only one methyl substitution on the benzene ring - i.e. lidocaine. However, no LA exists with just one chlorine substitution on the benzene ring. Therefore, applying this result requires acknowledging an important point. It can be inferred and conclusively decided that the additional substitutions in a clinically relevant LA, like chloroprocaine, must act to balance out the changes caused by a chlorine substitution on the benzene ring. The details of such a mechanism, however, is unclear.

\section{Conclusion}

In summary, comparisons between the four types of aromatic ring substituted RLAs yielded a host of results. Despite a greater spatial difference, benzene ring substitutions influenced LA effectiveness more than the type of intermediate linkage.

Substitutions on benzene ring were found to play a sizable role on the $\mathrm{N}_{\mathrm{t}}$ partial charge in quaternary LAs. In fact, benzene ring substitutions tended to favor a more conducive and negative partial charge and a lesser protonation propensity for an amino ester than an amino amide. Due to a lack of further testing, two exceptions occurred. The first exception happened with the addition of only a single methyl group to a LA. Further calculations are required to see if greater methylation of the benzene ring truly does lead to a greater effectiveness for amino esters. The other exception occured with the chlorination of the benzene ring in a LA. It is uncertain if greater chlorination emphasizes greater amide effectiveness over ester effectiveness; however, for the most part, the results support the WHO trend. Thus, it would benefit the LA design process to emphasize esters linkages over amide linkages.

However, a limitation of this computational study is that it can't definitively conclude whether one linkage system is superior to the other. The amide linkage may be more favored with different substitutions at different $\mathrm{R}$ positions. Theoretically, a definite conclusion could be reached through $\mathrm{SBDD}^{26}$. It would require an advanced design of this project.

A powerful computer would need to test all the possible combinations of substitution types and placements at the R positions for an amino ester in Fig. 1. Then, the computer would need to determine which combination leads to the greatest $\mathrm{N}_{\mathrm{t}}$ partial charge and a smallest protonation propensity. By limiting toxicity and molecular size under their maximum tolerable levels, it would then be possible to design a more effective, if not the most effective, LA that had existed.

For future theoretical experiments, I would hope to explore similar SBDD calculations for substitutions on the amide terminal; this would allow for a more inclusive look into $\mathrm{N}_{t}$ substituted LAs like bupivacaine ${ }^{33}$. Also, research to clarify how other electrophilic substitutions 
affect protonation propensities and $\mathrm{N}_{\mathrm{t}}$ partial charge would help to select more effective substitutions on the aromatic ring. Even though there exists a clear advantage for using aliphatic substitutions over electrophilic substitution, electrophilic substitutions are still seen in clinically useful LAs like benzocaine and tetracaine.

From this paper's' results, more ergonomic LA designs focusing on aromatic ring substitutions and choice of intermediate linkage can be targeted.

\section{Acknowledgments}

I thank Dr. Andrew Rappe and his teaching assistant Zeyu Zhou for guiding my progress in this paper. 


\section{References}

1. Strichartz, G. R., \& Ritchie, J. M. (1987). The Action of Local Anesthetics on Ion Channels of Excitable Tissues (pp. 21-52). https://doi.org/10.1007/978-3-642-71110-7_2

2. Becker, D. E., \& Reed, K. L. (2006). Essentials of local anesthetic pharmacology. Anesthesia Progress, 53(3), 98-108-10. https://doi.org/10.2344/0003-3006(2006)53[98:EOLAP]2.0.CO;2

3. Butterworth, J. F., \& Strichartz, G. R. (1990, April). Molecular mechanisms of local anesthesia: a review. Anesthesiology. https://doi.org/10.1097/00000542-199004000-00022

4. Bernardi, R. C., Gomes, D. E. B., Pascutti, P. G., Ito, A. S., Taft, C. A., \& Ota, A. T. (2007). Water solvent and local anesthetics: A computational study. International Journal of Quantum Chemistry, 107(7), 1642-1649. https://doi.org/DOI 10.1002/qua.21300

5. Hille, B. (1977). Local anesthetics: hydrophilic and hydrophobic pathways for the drugreceptor reaction. The Journal of General Physiology, 69(4), 497-515. https://doi.org/10.1085/jgp.69.4.497

6. Ragsdale, D. S., McPhee, J. C., Scheuer, T., \& Catterall, W. A. (1994). Molecular determinants of state-dependent block of $\mathrm{Na}+$ channels by local anesthetics. Science (New York, N.Y.), 265(5179), 1724-8. https://doi.org/10.1126/science.8085162

7. Bernardi, R. C., Gomes, D. E. B., Gobato, R., Taft, C. A., Ota, A. T., \& Pascutti, P. G. (2009). Molecular dynamics study of biomembrane/local anesthetics interactions. Molecular Physics, 107(14), 1437-1443. https://doi.org/10.1080/00268970902926238

8. Matsuki, H., Kaneshina, S., Kamaya, H., \& Ueda, I. (1999). Difference in surface activities between uncharged and charged local anesthetics. Anesthesia and Resuscitation, 35(1), 27-30. https://doi.org/10.1016/S0927-7765(98)00046-0

9. de Paula, E., \& Schreier, S. (1995). Use of a novel method for determination of partition coefficients to compare the effect of local anesthetics on membrane structure. Biochimica et Biophysica Acta, 1240(1), 25-33. https://doi.org/10.1016/0005-2736(95)00155-6

10. Hogberg, C.-J., \& Lyubartsev, A. P. (2008). Effect of Local Anesthetic Lidocaine on Electrostatic Properties of a Lipid Bilayer. Biophys. J., 94(2), 525-531. https://doi.org/10.1529/biophysj.107.104208

11. Wang, G.-K., \& Strichartz, G. R. (2012). State-Dependent Inhibition of Sodium Channels by Local Anesthetics: A 40-Year Evolution. Biochemistry (Moscow) Supplement. Series A, Membrane and Cell Biology, 6(2), 120-127. https://doi.org/10.1134/S1990747812010151

12. Lipkind, G., \& Fozzard, H. (2005). Molecular modeling of local anesthetic drug binding by voltage-gated sodium channels. Molecular Pharmacology, 1611-1622. https://doi.org/10.1124/mol.105.014803.of

13. Ragsdale, D. S., McPhee, J. C., Scheuer, T., \& Catterall, W. A. (1996). Common molecular determinants of local anesthetic, antiarrhythmic, and anticonvulsant block of 
voltage-gated Na+ channels. Proceedings of the National Academy of Sciences, 93(17), 9270-9275. https://doi.org/10.1073/pnas.93.17.9270

14. Marban, E., Yamagishi, T., \& Tomaselli, G. F. (1998). Structure and function of voltage-gated sodium channels. The Journal of Physiology, 508 ( Pt 3(Pt 3), 647-57. https://doi.org/10.1146/annurev.biochem.64.1.493

15. Seddon, A. M., Casey, D., Law, R. V., Gee, A., Templer, R. H., \& Ces, O. (2009). Drug interactions with lipid membranes. Chemical Society Reviews, 38(9), 2509. https://doi.org/10.1039/b813853m

16. Gupta, S. P. (1991). QSAR (quantitative structure-activity relationship) studies on local anesthetics. Chemical Reviews, 91(6), 1109-1119. https://doi.org/10.1021/cr00006a001

17. Eftink, M. R., Puri, R. K., \& Ghahramani, M. D. (1985). Local anesthetic-phospholipid interactions. The $\mathrm{pH}$ dependence of the binding of dibucaine to dimyristoylphosphatidylcholine vesicles. BBA - Biomembranes, 813(1), 137-140. https://doi.org/10.1016/0005-2736(85)90354-2

18. Bruce, A., Johnson, A., Lewis, J., Raff, M., Roberts, K., \& Walter, P. (2002). The lipid bilayer. In Molecular Biology of the Cell. Garland Science. Retrieved from https://www.ncbi.nlm.nih.gov/books/NBK26871/

19. Rosenberg, P., Veering, B., \& Urmey, W. (2004). Maximum recommended doses of local anesthetics: A multifactorial concept. Regional Anesthesia and Pain Medicine, 29(6), 564-575. https://doi.org/10.1016/j.rapm.2004.08.003

20. Malheiros, S. V. P., Pinto, L. M. A., Gottardo, L., Yokaichiya, D. K., Fraceto, L. F., Meirelles, N. C., \& De Paula, E. (2004). A new look at the hemolytic effect of local anesthetics, considering their real membrane/water partitioning at $\mathrm{pH}$ 7.4. Biophysical Chemistry, 110(3), 213-221. https://doi.org/10.1016/j.bpc.2004.01.013

21. Wolf, A. R., Valley, R. D., Fear, D. W., Roy, W. L., \& Lerman, J. (1988). Bupivacaine for caudal analgesia in infants and children: the optimal effective concentration. Anesthesiology, 69(1), 102-6. Retrieved from http://www.ncbi.nlm.nih.gov/pubmed/3291644

22. Krahl, M. E., Keltch, A. K., \& Clowes, G. H. A. (1940). The role of changes In Extracellular and Intracellular Hydrogen Ion Concentration in the action of local anesthetic bases. Journal of Pharmacology and Experimental Therapeutics, 68(3).

23. Haas, D. A. (2002). An Update on Local Anesthetics in Dentistry ( Mise à jour sur les anesthésiques locaux utilisés en dentisterie ). Journal of the Canadian Dental Association, 68(9), 546-551. https://doi.org/10.1034/j.1601-1546.2002.10103.x

24. World Health Organization. (2015). WHO Model List of Essential Medicines - 19th List (April 2015). Essential Medicines, (April), 1-45. https://doi.org/10.1016/S1473-3099(14)70780-7

25. Yu, W., \& Mackerell, A. D. (2017). Computer-aided drug design methods. In Methods in Molecular Biology (Vol. 1520, pp. 85-106). Humana Press, New York, NY. https://doi.org/10.1007/978-1-4939-6634- 
26. Catterall, W. a. (2000). From Ionic Currents to Molecular Mechanisms : The Structure and Function of Voltage-Gated Sodium Channels. Neuron, 26(1), 13-25. https://doi.org/10.1016/S0896-6273(00)81133-2

27. Ohno, K., Kamiya, N., Asakawa, N., Inoue, Y., \& Sakurai, M. (2001). Application of an integrated MOZYME+DFT method to pKa calculations for proteins. Chemical Physics Letters, 341(3-4), 387-392. https://doi.org/10.1016/S0009-2614(01)00499-7

28. Xiang, T. X., \& Anderson, B. D. (1994). The relationship between permeant size and permeability in lipid bilayer membranes. The Journal of Membrane Biology, 140(2), 111-122. https://doi.org/10.1007/BF00232899

29. Lee-Son, S., Wang, G. K., Concus, A., Crill, E., \& Strichartz, G. (1992). Stereoselective inhibition of neuronal sodium channels by local anesthetics. Evidence for two sites of action? Anesthesiology. Retrieved from http://www.ncbi.nlm.nih.gov/pubmed/1322642

30. Ritchie, J. M., \& Greenard, P. (1966). On the mode of action of local anesthetics. Annual Review of Pharmacology and Toxicology, 6, 405-430. Retrieved from http://www.annualreviews.org/doi/pdf/10.1146/annurev.pa.06.040166.002201

31. Galindo, A., \& Witcher, T. (1980). Mixtures of local anesthetics: bupivacaine-chloroprocaine. Anesthesia and Analgesia, 59(9), 683-5.

32. Feldman, H. (1988). Comparative motor-blocking effects of bupivacaine and ropivacaine, a new amino amide local anesthetic, in the rat and dog. Journal of Anesthesia and Analgesia, 67(11), 1047-1055.

33. Galindo, A., \& Witcher, T. (1980). Mixtures of local anesthetics: bupivacaine-chloroprocaine. Anesthesia and Analgesia, 59(9), 683-5. 